\title{
Principles of response determination: The list-rule model of SR compatibility
}

\author{
THIERRY HASBROUCQ, YVES GUIARD, and LYDIE OTTOMANI \\ Centre National de la Recherche Scientifique, Marseilles, France
}

(Bertram Scharf, Sponsor)

\begin{abstract}
In this theoretical note, we address the issue of stimulus-response compatibility (SRC). We propose an operational definition of the SRC variable and draw some implications of this definition for cognitive modeling. We also outline a general model of the controlled processes of response determination that accounts for the SRC phenomena reported in the literature.
\end{abstract}

\section{THE NOTION OF STIMULUS-RESPONSE COMPATIBILITY AND ITS IMPLICATIONS}

\section{Defining Stimulus-Response Compatibility}

One of the most reliable findings of human information processing research is that the manipulation of the relationship between the stimulus $(S)$ and the response $(R)$ has quite powerful effects on reaction time (RT) (e.g., Fitts \& Deininger, 1954; Fitts \& Seeger, 1953). To refer to this class of effects, Fitts and his collaborators introduced the term stimulus-response compatibility (SRC), which has since become accepted through use. Until recently (Kornblum, Hasbroucq, \& Osman, 1984, in press), SRC has often been considered a set of unrelated empirical facts, which consequently have not needed to be formalized as a whole. The fragmented approach that characterizes the SRC literature is probably due to the fact, pointed out by Sanders (1980), that most of the definitions of SRC that have been proposed since the 1950s are in some sense circular. For instance, Broadbent and Gregory (1965) stated that "responses which bear a natural or 'compatible' relation to their appropriate stimuli tend to be faster than responses which are less natural or more "incompatible'" (p. 61). In other words, performance rapidity is supposed to be explained by SRC, and it is suggested that the defining criterion for SRC is precisely performance rapidity. Such vague characterizations of the SRC variable have generally been felt sufficient by investigators incidentally interested in SRC, as there is some convergence among individual intuitive representations with respect to what is meant by a "compatible" SR arrangement (cf. Fitts's, 1951, concept of a population stereotype). Nonetheless, it is obvious that to study the SRC issue per se, one cannot bypass the problem of an appropriate starting definition. Thus, we propose to

T. Hasbroucq and Y. Guiard are in the Unité de Neurosciences Cognitives, and L. Ottomani is with the Equipe de Modélisation des Deplacements Orientés of the CNRS. During the preparation of this paper, the first author was supported by ONR Grant N0001489-J-1557. Correspondence may be sent to T. Hasbroucq, CRNS-LNF1, 31 Che$\min$ J. Aiguier, 13402 Marseille Cedex 9, France. define an SRC effect as the modification of performance induced by a change in the SR relationship that is uncorrelated with any change in stimulation or responding. This definition, implicitly shared by some authors (e.g., Inhoff, Rosenbaum, Gordon, \& Campbell, 1984; Kornblum et al., 1984, in press), has two main implications that are developed below.

\section{Procedural Variations of \\ Stimulus-Response Compatibility}

First, it must be noted that the above definition implies no supposition whatsoever on the explanatory mechanisms of SRC: It is strictly operational in the sense that it refers only to the procedures that may allow the empirical testing of hypotheses on SRC. There are two such procedures, both of which have been identified by Fitts and his coworkers.

The first one consists simply of varying, by merely changing the instructions, the mapping of the $S$ set onto the $R$ set (Fitts \& Deininger, 1954). Suppose, for example, that $S$ is a positional random variable with two values, left and right, and the values chosen for $R$ are also left and right. One may ask the subject to perform either a direct, ipsilateral SR transformation or an inverted, contralateral SR transformation. The difference that will almost inevitably emerge in RT in favor of the former mapping condition will unambiguously be interpretable as an SRC effect, as the manipulation of SR mapping allows a change in the SR relationship to the exclusion of any change in the $S$ or $R$ domain.

The second procedure that allows the testing of hypotheses on SRC consists of varying the match formed by the variables $S$ and $R$, independently of the mapping. This procedure necessitates the recourse to several $S$ variables and several $R$ variables, at a constant level of uncertainty, and it is through an exploration of their combinations that SRC can be assessed (Fitts \& Seeger, 1953). Thus, for example, suppose that, as in Hasbroucq and Guiard $(1988,1990)$, four conditions are created by using either position (left/right) or color (red/green) as the $S$ variable, and either position (left/right) or color 
(red/green) as the $R$ variable. In two conditions (positionto-position and color-to-color SR transformations), the $S$ and $R$ variables will match each other; in the two others (position-to-color and color-to-position SR transformations), there is no match between $S$ and $R$. With such protocols, the average RT is typically shorter in the two conditions in which the $S$ and $R$ variables match each other (Hasbroucq \& Guiard, 1988, 1990).

The two above-described experimental procedures allow the experimenter to clearly see changes in performance related to changes in the SR relationship per se and, thus, are consistent with our definition of SRC. Note that, according to current opinion, a third procedure introduced by Simon and Rudell (1967) and extensively used in the literature would allow an SRC contrast. However, Hasbroucq and Guiard $(1988,1990)$ have recently demonstrated that this paradigm cannot be utilized in the study of SRC: It is severely flawed in that it confounds the effect of an irrelevant spatial correspondence between $S$ and $R$ with that of $S$ congruity, the correspondence relationship borne by two simultaneous aspects of the $S$. In a series of experiments, Hasbroucq and Guiard have brought decisive evidence that $S$ congruity accounts, in fact, for the whole of the so-called "Simon effect." From these studies, one can draw the conclusion that the Simon effect is in fact a spatial variant of the Stroop effect and is therefore irrelevant to the SRC issue. For this reason, we will deliberately ignore the Simon effect in this paper.

\section{Stimulus-Response Compatibility and the \\ Response Determination Stage}

The second implication of the above definition concerns the relationship between SRC and information processing stages. To define SRC, one has to reason about three entities, the $S$, the $R$, and the SR relationship; therefore, a minimal three-stage decomposition of choice RT is necessary to localize the effects of SR match and SR mapping in the information-processing chain. One has simply to assume that the system first identifies the $S$ (i.e., produces the mental symbol $s$ corresponding to the physical event $S$ ), then performs the abstract sensory-motor transformation (i.e., retrieves the mental symbol $r$ corresponding, by virtue of the instructions, to the mental symbol $s$ ), and finally programs and executes the $R$ (i.e., produces the physical event $R$ specified by $r$ ). Within this framework, the locus of the SR mapping effect and SR match effect is the central processing stage $-R$ determination in Theios's (1975) terminology. Note that this analysis is well supported by converging evidence accumulated with the help of Sternberg's (1969) additive factor method, which has generally shown the additivity of SRC factors with perceptual factors (e.g., Hasbroucq, Guiard, \& Kornblum, 1989) and motor factors (e.g., Hasbroucq et al., 1989). Thus, the problem of understanding the mental mechanisms of SRC amounts to that of understanding how the two SRC factors identified above (SR mapping and SR match) coordinate their effects on the operations taking place at the stage of $R$ determination.

\section{MODELING THE RESPONSE DETERMINATION PROCESSES}

\section{The Concept of Dimensional Overlap}

A first step has been recently made by Kornblum and his collaborators (Kornblum et al., 1984, in press), who elaborated a systematic account of the articulation of the effects of SR mapping and SR match on information processing. Kornblum et al.'s account of SRC was based on the concept of dimensional overlap (DO) between $S$ and $R$ sets, a continuous representational variable characterizing the SR relationship at the level of SR match. The effect of SR mapping, the authors proposed, should be considered as the metric of the DO between the $S$ and $R$ sets. That is to say, the effect of SR mapping will emerge in a given task to the extent that there is DO between the variables $S$ and $R$.

The functional, real-time mechanism postulated by Kornblum et al. to account for the effects of SR match and SR mapping is an automatic activation process. According to Kornblum et al., when the $S$ and $R$ sets have DO, elements in the $S$ set automatically activate corresponding elements in the $R$ set. When the automatically activated $R$ element is the one required by the instructions, the RT is fast. In contrast, when the automatically activated element is not the one required, the RT is slower. As pointed out by Hasbroucq and Guiard (1988), in this model, the assumption of an automatic activation is only necessary to account for the Simon effect. Such an assumption appears to be pointless from the moment thatfor the reasons developed by Hasbroucq and Guiard (1988)-one ceases to take the Simon effect into consideration for modeling the mechanisms of SRC. Thus, in our opinion, if the representational component of the DO model (the DO concept proper) is promising and well supported by available evidence, its functional mechanisms should be revised. In keeping with the DO concept, one of us (Hasbroucq, 1987) has proposed an alternative model of the real-time processes that might occur during the $R$ determination stage. In contrast with the automatic activation hypothesis, this tentative theoretical account of SRC is elaborated under the assumption that SRC effects only reflect changes in the speed of controlled SR transformation operations, that is, without having to resort to an uncontrolled, automatic process at the $R$ determination stage.

\section{Cognitive Mechanisms: Memory-List Scanning and Rule Implementation}

It must be pointed out that, depending on whether or not the $S$ and $R$ variables form a match at an abstract, conceptual level (i.e., if they have DO), the set of cognitive mechanisms available to the subject for the completion of the $R$ determination operation must drastically differ. In essence, our suggestion is that $R$ determination may take one of two prototypic forms: either memorylist scanning or rule implementation. The virtues and the constraints of these two forms are complementary. The 
virtue of the memory-list scanning operation is that it allows an individual to perform any SR transformation task, making it possible, in particular, to succeed in the complete absence of DO. This universally applicable solution to the SR transformation problem comes up, however, against the constraint of short-term memory limitations. Conversely, the rule-implementation form of the SR transformation operation is only applicable in tasks in which the variables $S$ and $R$ can be represented in terms of the same concept (i.e., in the presence of DO). In return, this form has the virtue of being insensitive to the limits of short-term memory. As a shorthand, we will call this model the list-rule model (see Figure 1).

Suppose that, for the subject, the $S$ and $R$ variables correspond to altogether different concepts, as was presumably the case, for example, in one condition of Fitts and Deininger's (1954) experiment in which the $S$ was drawn from a set of proper names and the $R$ was a spatially oriented movement. For such a task, characterized by the impossibility to mentally represent the SR transformation task in terms of any algorithm of the form $r=f(s)$ (Duncan, 1977a, 1977b; Shaffer, 1965, 1966), the only mechanism conceivably available to the subject at the $R$ deter- mination stage is the (probably serial) exploration of a memorized list of individual $s r$ pairs. Under this hypothesis, one expects no effect of SR mapping on RT, but a strong effect of the number of SR alternatives, since the length of the hypothetical list will be a major determinant of the duration of the $R$ determination process.

Now suppose, alternatively, that conceptually the variables $S$ and $R$ do form a match for the subject: for example, the $S$ is drawn from a set of $N$ colors, and the $R$, likewise, consists of the designation of a color among the same set. Two cases must be distinguished. If the instructions require an irregular, arbitrarily defined color-to-color transformation (e.g., to indicate blue in response to yellow, red in response to blue, etc.), it is clear that no systematic SR transformation will be accessible to the subject. Thus, a memory-list scanning procedure will presumably have to take place at the $R$ determination stage, as in a task with no SR match. If, alternatively, the required SR transformation is formulated by the experimenteror at least understood by the subject-as a systematic transformation rule, then one need not postulate a listscanning procedure at the stage of $\boldsymbol{R}$ determination. Rather, it is reasonable to assume that the subject par-

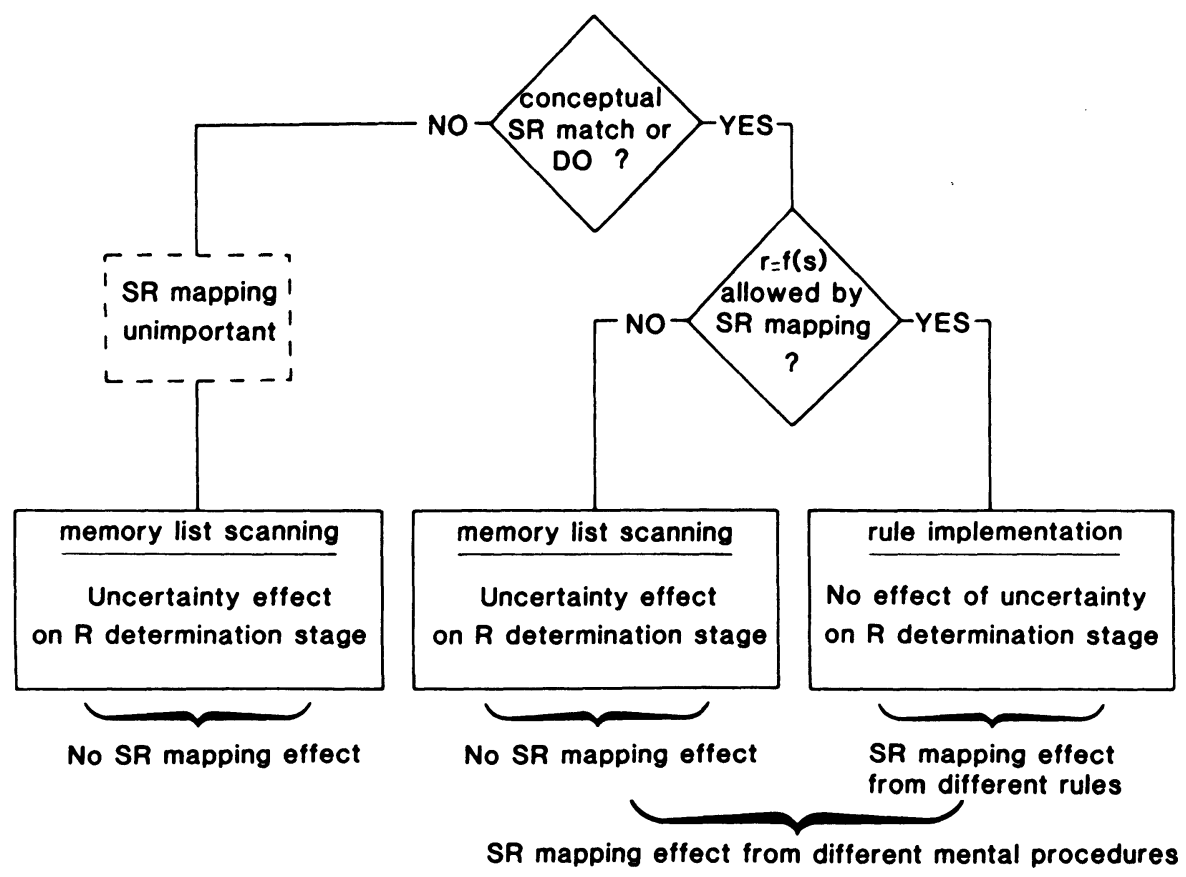

Figure 1. The list-rule model of $R$ determination. If the variables $S$ and $R$ cannot be represented in terms of a common concept, the only $R$ determination procedure conceivably available to the subject during RT will consist of scanning the memorized list of the individual $s r$ pairs that have been defined in the instructions as correct. In this case, no effect of SR mapping is predicted, but one expects the number of SR alternatives to affect the duration of the $R$ determination stage. If, alternatively, the subject does reduce the variables $S$ and $R$ to the same concept, the possibility of an algorithmically governed $R$ determination procedure will emerge. This possibility, however, will not be necessarily exploitable: If the prescribed SR mapping prohibits the formulation of a rule, the subject will be forced to retreat to the list-scanning $R$ determination procedure; if, alternatively, the SR mapping is representable as a rule-and so represented actually by the subject-he/she will be able to save memory and implement, at the stage of $R$ determination, a memorized algorithm of the form $r=f(s)$. In the latter case, no effect of number of alternatives is predicted on the $R$ determination stage (that is, the effect of this factor is expected to be relatively weak on RT), but one does expect an effect of SR mapping on RT on the grounds that the implementation of different rules may take different times at the stage of $R$ determination. 
simoniously makes use of the memorized rule, that is, determines the mental symbol $r$ on the basis of the available algorithm $r=f(s)$.

The predictions, thus, will differ in the case of SR match, or DO, according to whether or not the possibility of a systematic, algorithmically governed transformation is offered to-and actually exploited by-the subject. If no systematic SR transformation rule can be brought into play during task performance, the predictions are the same as in the case where there is no SR match. If, however, the subject is allowed to carry out a systematic SR transformation, one predicts the possibility of an SR mapping effect, which will reflect the difference in the time required for the implementation of different rules (in the particular case of a two-choice reaction task, the mapping effect is interpretable as the indication that an identical and an alternate SR transformation operation take different times at the $R$ determination stage). Second, one must predict the disappearance of the effect of the number of alternatives on the $\mathbf{R}$ determination stage since the time to complete an operation of the form $r=f(s)$ should remain constant, regardless of the probability of $S$ and $R$. Therefore, one predicts a reduction of the size of the uncertainty effect on RT on the grounds that one of the three loci of this effect (in the minimal three-stage model) has become insensitive to it. However, this does not imply a complete disappearance of the uncertainty effect on RT, since the initial and final stages of the informationprocessing chain may still be influenced by uncertainty.

Thus, the list-rule model of $R$ determination accounts for the fact that an SR mapping effect does or does not occur in a given choice reaction task in terms that rely on the DO concept. The rationale, however, differs from that offered by Kornblum et al. (1984, in press) in two notable respects. As we have pointed out, the first, obvious, difference is that with the list-rule model one need not suppose that an $S$ automatically activates an $R$ in order to explain the mapping effect in the presence of DO. The second, more subtle, difference lies in the assumption inherent to the list-rule model that, in a task characterized by DO between the $S$ and $R$, the mapping effect may emanate from one of two sources (see Figure 1). A mapping effect may result either from the fact that in different conditions the subject has recourse to altogether different procedures (memory-list scanning vs. rule implementation) or, in the case in which the subject may carry out systematic SR transformations, from the fact that he/she uses different rules. For example, we may reasonably imagine that, in a two-choice RT task with DO, reversing the SR mapping will have the effect of forcing the subject not to resort to a memory-list scanning procedure, but rather to use an inverted SR transformation rule likely to require more time than a direct one at the stage of $R$ determination, since its implementation necessitates the inversion of the mental symbol first produced by the logically minimal transformation-the identical $(r=s)$ rule.

The list-rule model of SRC, a descendant of the DO model, seems consistent with available empirical evidence, but it is still in an early phase of its development.
Obviously, more research is needed to evaluate its validity in some detail. However, we feel that we can safely say that research on SRC represents a typical instance of research on the voluntary, controlled mechanisms of cognition.

\section{REFERENCES}

Broadbent, D. E., \& Gregory, M. (1965). On the interaction of S-R compatibility with other variables affecting reaction time. British Journal of Psychology, 56, 61-67.

Duncan, J. (1977a). Response selection errors in spatial choice reaction tasks. Quarterly Joumal of Experimental Psychology, 29, 415-423.

Duncan, J. (1977b). Response selection rules in spatial choice reaction tasks. In S. Dornic (Ed.), Attention and performance VI (pp. 4961). Hillsdale, NJ: Erlbaum.

FIrTs, P. M. (1951). Engineering psychology and equipment design. In S. S. Stevens (Ed.), Handbook of experimental psychology (pp. 12871340). New York: Wiley.

FITTS, P. M., \& DeInINGer, R. L. (1954). S-R compatibility: Correspondence among paired elements within stimulus and response codes. Journal of Experimental Psychology, 48, 483-491.

FitTs, P. M., \& Seeger, G. M. (1953). S-R compatibility: Spatial characteristics of stimulus and response codes. Journal of Experimental Psychology, 46, 199-210.

HasbroucQ, T. (1987). Contribution à l'étude de la compatibilité stimulus-réponse: essai de formalisation théorique, résultats expérimentaux. Unpublished doctoral dissertation, Université de Provence, Marseille, France.

Hasbrouce, T., \& GuIARD, Y. (1988, January). Congruence du stimulus et compatibilité stimulus-réponse: l'effet Simon relève du domaine perceptif. Paper presented at the meeting on "Automatisme et Contrôle" of the Société Française de Psychologie, Dijon, France.

HASBRoucQ, T., \& GuIARD, Y. (1990). Irrelevance of the Simon effect to the issue of stimulus-response compatibility: Towards a conceptual clarification. Manuscript submitted for publication.

Hasbrouce, T., Guiard, Y., \& Kornblum, S. (1989). The additivity of stimulus-response compatibility with the effects of sensory and motor factors in a tactile choice reaction time task. Acta Psychologica, 72, 139-144.

Inhoff, A. W., Rosenbaum, D. A., Gordon, A. M., \& Campbell, J. A. (1984). Stimulus-response compatibility and motor programming of manual response sequences. Journal of Experimental Psychology: Human Perception \& Performance, 10, 724-733.

Kornblum, S., HasbroucQ, T., \& Osman, A. (1984, November). The dimensional overlap model of stimulus-response compatibility. Paper presented at the annual meeting of the Psychonomic Society, San Antonio, TX.

Kornblum, S., HasbroucQ, T., \& Osman, A. (in press). Dimensional overlap-Cognitive basis for stimulus-response compatibility: A model and taxonomy. Psychological Review.

SANDERS, A. F. (1980). Stage analysis of reaction processes. In G. E. Stelmach \& J. Requin (Eds.), Tutorials in motor behavior (pp. 331354). Amsterdam: North-Holland.

ShAFFER, L. H. (1965). Choice reaction with variable S-R mapping. Journal of Experimental Psychology, 70, 284-288.

ShafFer, L. H. (1966). Some effects of partial advance information on choice reaction with fixed or variable S-R mapping. Journal of Experimental Psychology, 72, 541-545.

Simon, J. R., \& RUDELl, A. P. (1967). Auditory S-R compatibility: The effect of an irrelevant cue on information processing. Journal of Applied Psychology, 51, 300-304.

STERNBERG, S. (1969). The discovery of processing stages: Extensions of Donders' method. In W. G. Koster (Ed.), Attention and performance II (pp. 276-315). Amsterdam: North-Holland.

Theios, J. (1975). The components of response latency in simple human information processing tasks. In P. M. A. Rabitt and S. Dornic (Eds.), Attention and performance $V$ (pp. 418-440). New York: Academic Press.

(Manuscript received December 5, 1989.) 